\title{
Pyruvate kinase M2 fuels multiple aspects of cancer cells: from cellular metabolism, transcriptional regulation to extracellular signaling
}

Ming-Chuan $\mathrm{Hsu}^{1}$ and Wen-Chun Hung ${ }^{1,2^{*}}$

\begin{abstract}
Originally identified as a metabolic enzyme that catalyzes the transfer of a phosphate group from phosphoenolpyruvate (PEP) to ADP in the glycolytic pathway, pyruvate kinase M2-type (PKM2) has been shown to exhibit novel biological activities in the nucleus and outside the cells. Although cell-based studies reveal new non-canonical functions of PKM2 in gene transcription, epigenetic modulation and cell cycle progression, the importance of these non-canonical functions in PKM2-mediated tumorigenesis is still under debate because studies in genetically modified mice do not consistently echo the findings observed in cultured cancer cells. In addition to regulation of gene expression, the existence of PKM2 in exosomes opens a new venue to study the potential role of this glycolytic enzyme in cell-cell communication and extracellular signal initiation. In this review, we briefly summarize current understanding of PKM2 in metabolic switch and gene regulation. We will then emphasize recent progress of PKM2 in extracellular signaling and tumor microenvironment reprogramming. Finally, the discrepancy of some PKM2's functions in vitro and in vivo, and the application of PKM2 in cancer detection and treatment will be discussed.
\end{abstract}

\section{Background}

Biochemical analysis by charactering the enzymatic activity that catalyzes the formation of lactate from glucose in cell lysates revealed the first intracellular metabolic pathway, the glycolytic pathway. Beginning from the purification of fractions that contained glycolytic activity, a number of pioneer researchers contributed to the identification of enzymes that involve in each step in the pathway [1-3]. These results build up our modern concept in the interchange of aerobic and anaerobic respiration and energy production under various physiological and pathological circumstances.

The existence of an enzyme that catalyzed the production of ATP by transferring a phosphate group from PEP to ADP in the liver was first reported in 1934 [4]. Subsequent isolation of the enzyme, known as pyruvate kinase (PK) later, demonstrated differences in tissue distribution

\footnotetext{
* Correspondence: hung1228@nhri.org.tw

${ }^{1}$ National Institute of Cancer Research, National Health Research Institutes, No. 367, Shengli Road, Tainan 704, Taiwan

${ }^{2}$ Institute of Medicine, College of Medicine, Kaohsiung Medical University, Kaohsiung 802, Taiwan
}

and catalytic kinetics suggesting this enzyme may have different isoforms [5-8]. During 1982 to 1984, various PK genes were cloned from yeast, chicken and rat [912]. The functional study of PKM2 was initiated by the identification of a candidate gene in mouse in early 1980s [11]. Later, Noguchi et al. showed that two (12]. In human, PKM isoforms are also produced via a similar splicing PKM2 mRNA separately [13].

Several findings caught researcher's attention to the potential role of PKM2 in tumorigenesis. First, PKM2 is the embryonic isoform that highly expressed during development. Its transcription is attenuated in a $[14,15]$. Second, study of the relative abundance of PKM1 and PKM2 in normal and tumor tissues demonstrated a switch from the PKM1 isoform to the PKM2 isoform in various cancers like hepatocellular carcinoma $[16,17]$. Third, the change of mRNA splicing from 
PKM1 to PKM2 is enhanced by c-Myc oncogene suggesting cancer cells actively engage in this switch to fit their requirement in proliferation and metabolism [18]. Fourth, modulation of PKM2 activity by activators or inhibitors affect tumor growth in vivo [19-21].

\section{The first episode: PKM2 as a metabolic enzyme in the cytoplasm}

Since the role of PKM2 in metabolic control of glycolysis in cancer cells has been extensively reviewed [22-24], we only summarize three crucial differences between PKM1- and PKM2-mediated catalysis and cellular metabolism here. The first difference is subunit interaction. Both PKM1 and PKM2 are tetrameric proteins formed by four identical subunits. Each subunit (or monomer) contains four structural domains including $\mathrm{A}, \mathrm{B}, \mathrm{C}$, and $\mathrm{N}$-terminal domain. The monomer first dimerizes together and then two dimers interact via the dimer-dimer interface orchestrated by the $\mathrm{C}$ domain of monomer to form a tetramer. Because PKM1 and PKM2 include different exons in their mRNAs, this changes the encoded amino acids in the $\mathrm{C}$ domain and alters the tetramer stability. Under physiological condition, PKM1 constitutively organizes as a tetramer while PKM2 can be existed in tetramer or dimer. The second difference is allosteric regulation. Depending on the intracellular concentrations of small molecules and metabolites, the activity of PKM1 and PKM2 can be differentially regulated. One of the most well-known allosteric regulators is fructose-1,6-bisphosphate (FBP). This glycolytic intermediate directly binds PKM2 and increases the affinity of PKM2 for PEP [25]. On the contrary, FBP does not significantly affect PKM1 activity. In addition to FBP, other metabolites, amino acid and small molecules have been reported to affect PKM2 activity [19, 26-31] (Fig. 1). However, the concentration required for activation or inhibition is high and the modulatory effect is modest. Whether these small molecules play an important role in the control of PKM activity under physiological circumstances is still unclear. PKM2 activity is also regulated by post-

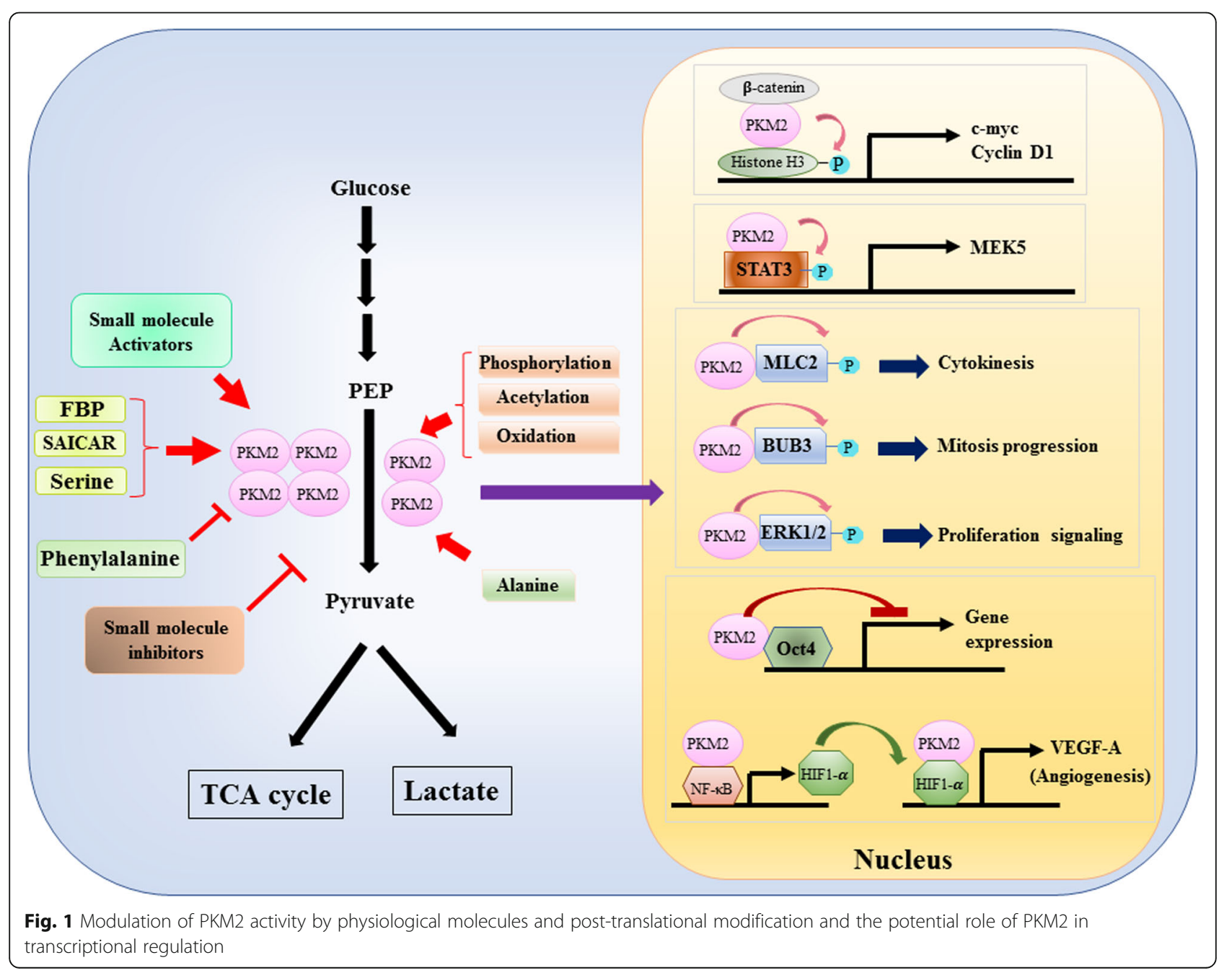


translational modification, such as phosphorylation, acetylation and oxidation, which favor the low activity of dimeric PKM2 (Fig. 1). The third difference is energy production and intermediate utilization. Since PKM1 constitutively exists as the active tetramer, the main biological function of this isoform is the generation of ATP to supply cellular energy. However, PKM2, in addition to produce ATP, can switch to the less active dimeric form to generate several glycolytic intermediates which can be used as building blocks for the biosynthesis of amino acids, lipids and nucleotides.

\section{The second episode: PKM2 as a signaling modulator in the cytoplasm}

In addition to function as a glycolytic enzyme, PKM2 is proposed to involve in more cellular processes due to the identification of interacting proteins in the cytoplasm. For example, PKM2 was shown to be an interacting protein of several tyrosine kinases including A-Raf, Break point cluster region-Abelson (BCR$\mathrm{ABL}$ ) fusion kinase, fibroblast growth factor receptor 1 (FGFR1) etc. [32, 33]. These binding partners have been shown to modulate the dimeric/tetrameric change of PKM2 to alter cell metabolism. However, it is possible that PKM2 may reciprocally affect the catalytic kinetics, substrate binding and cytoplasmic location of these binding partners to modulate signal transduction. The finding that PKM2 is a phosphortyrosine binding protein strengthens this possibility because many intracellular signaling mediators can bind to phosphor-tyrosine residue to assemble specific protein complexes for signal transmission [34]. To date, the list of PKM2 binding partners grows continuously. We highlight several new members and discuss their biological implication here. Mukheriee et al. demonstrated that PKM2 could bind with $\mathrm{HuR}$, a RNA binding protein which plays an important role in the control of mRNA stability and translational efficiency, to promote cell cycle progression and proliferation of glioma cells [35]. Interestingly, another RNA binding protein tristetraprolin which could bind a number of mRNA via the AU-rich element at $3^{\prime}-$ untranslational region (3'-UTR) was also found to be a PKM2 interacting partner, and PKM2 induced phosphorylation and degradation of tristetraprolin to modulate breast cancer growth [36]. These two studies imply a potential translational control function of PKM2. Recently, Liang et al. identified the antiapoptotic protein Bcl2 as a new PKM2 partner [37]. They demonstrated that oxidative stress induced the translocation of PKM2 into mitochondria where it phosphorylated and stabilized $\mathrm{Bcl} 2$ by preventing its degradation via ubiquitination-dependent pathway. These data suggested that PKM2 helps cancer cells to adapt oxidative stress elicited by intracellular metabolic change or extracellular insult.

\section{The third episode: PKM2 as a transcriptional regulator in the nucleus}

A nuclear role of PKM2 in the regulation of gene transcription or epigenetic modification was firstly suggested by the finding that PKM2 bound with Y333phosphorlated $\beta$-catenin, and the $\beta$-catenin-PKM2 complex was recruited to the nucleosomes to phosphorylate histone H3 at threonine 11 [38] (Fig. 1). This phosphorylation subsequently increased histone $\mathrm{H} 3$ acetylation that led to upregulation of $\beta$-catenin target genes. Another transcription factor directly phosphorylated by PKM2 is signal transducer and activator of transcription 3 (STAT3) [39]. PKM2-mediated phosphorylation of STAT3 at tyrosine 705 enhanced STAT3 activity to upregulate the expression of mitogen-activated protein kinase kinase 5 (MEK5). Beside transcription factors, PKM2 has been shown to phosphorylate myosin light chain 2 (MLC2), BUB3 and extracellular signal-regulated kinase 1 and 2 (ERK1 and ERK2) [40-42]. Interestingly, PKM2 also acts via phosphorylation-independent manner to affect gene expression. For example, PKM2 has been found to bind with Oct4, one of the master transcription factors that control self-renewal of stem cells, and inhibit Oct4-mediated transcription [43]. PKM2 can also enhance tumor angiogenesis by interacting with NF- $\kappa B$ and HIF- $1 \alpha$ in the nucleus and activating the expression of HIF- $1 \alpha$ target gene VEGF-A. Consequently, increased secretion of VEGF-A boosts blood vessel formation which contributes to tumor growth [44]. Although these studies strongly suggested the nuclear localization and protein kinase function of PKM2 in various physiological and pathological circumstances, however the importance of nuclear PKM2-mediated gene expression has been challenged by studies using PKM2 knockout cells. By using $\left[{ }^{32} \mathrm{P}\right]$-labeled PEP and PKM2-null mouse embryonic fibroblasts, Hosios et al. showed that PEP-dependent phosphorylation is not a common event in cells and the reaction is not catalyzed by PKM2 [45]. The discrepancy of these studies is currently unresolved and the protein kinase activity of PKM2 needs further confirmation.

\section{The fourth episode: PKM2 as an extracellular signaling communicator}

The presence of extracellular PKM2 opens a new avenue for the study of PKM2 biological function. Buschow et al. provided the first evidence that PKM2 could be detected in B-cell exosomes and was identified as a MHC class II-associated protein [46]. Two subsequent studies also indicated that PKM2 is existed in exosomes released by various cells $[47,48]$. Currently, several 
public databases like ExoCarta and EVpedia provide comprehensive information for the components including proteins, lipids, nucleic acids of extracellular vesicles in different species. All of the data confirm that PKM2 is a package protein of exosomes. Recent studies have clearly demonstrated a communicative role of exosomes by delivering different components from host cells to recipient cells [49-51]. It is expectable that PKM2 may play a role in cell-cell crosstalk.

Emerging evidence indeed support this hypothesis. For example, a recent study demonstrated that blood circulating PKM2 may promote tumor growth and angiogenesis by increasing the growth, migration and matrix adhesion of endothelial cells [52]. Another investigation also showed that PKM2 secreted from colon cancer cells might act via an autocrine stimulation to enhance cell migration by activating the PI3K/Akt and Wnt/ $\beta$-catenin pathways [53]. In addition to cancer cells, neutrophils at the tissues damage sites could release PKM2 to promote angiogenesis and wound healing [54]. Our recent study also demonstrated that recombinant PKM2 protein could induce phosphorylation and activation of epidermal growth factor receptor (EGFR) [55]. Moreover, we found that R339E mutant PKM2 which preferentially formed dimeric PKM2 activated EGFR more significantly than the tetrameric PKM2. Keller et al. identified 154 proteins as potential substrates for PKM2 after treatment of Hela cells with succinyl-5-aminoimidazole-4carboxamide-1-ribose- $5^{\prime}$-phosphate (SAICAR), an intracellular metabolite which could stimulate the protein kinase activity of PKM2 [42]. They also found EGFR as a PKM2 substrate. Their results are different from ours in two ways. First, the signaling pathways activated in our study are elicited by extracellular PKM2 while the molecular targets identified in their study are potential substrates of intracellular PKM2. Second, increase of ERK1/2 activity in our study is initiated by EGFR activation while ERK1/2 activation in their study is directly stimulated by the SAICAR/PKM2 complex. One similar phenomenon observed in both studies is that R339E mutant PKM2 activates signaling molecules more significantly than the wild type PKM2 suggesting the distinct role of dimeric and tetrameric PKM2 in oncogenesis. By using receptor tyrosine kinase array, we found that extracellular PKM2 only activated limited growth factor receptors in breast cancer cells (data not shown). Currently, the selectivity of receptor activation by extracellular PKM2 remains unknown. In addition, why R339E mutant PKM2 is more potent in the activation of EGFR is also not clear. More experiments are needed to answer these questions.

Another elegant question to be addressed is whether free PKM2 and vesicle-packaging PKM2 exhibit similar effect in promoting tumorigenesis (Fig. 2). Free extracellular
PKM2 could not penetrate plasma membrane and could only activate intracellular signaling via cell surface proteins like growth factor receptors. Conversely, vesiclepackaging PKM2 could be endocytosed by cancer and stroma cells, and the PKM2 released from vesicles could affect metabolism and gene expression via intracellular mechanism. The PKM2-null cells or mice will be useful to elucidate whether these two extracellular forms of PKM2 could act synergistically or antagonistically in tumorigenesis.

\section{The fifth episode: unanswered discrepancy of PKM2}

In addition to the cell-based data discussed above, the oncogenic role of PKM2 has also been challenged after the generation of PKM2 knockout mice. Israelsen et al. generated a conditional knockout mouse model by deleting the PKM2-specific exon 10 [56]. Surprisingly, depletion of PKM2 accelerated but not attenuated tumor formation driven by loss of Brcal gene in mice. These data indicated that PKM2 is not required for the proliferation of cancer cells. Interestingly, PKM1 expression was only detected in non-proliferating tumor cells suggesting a tumor-suppressive role of PKM1 in breast cancer. In addition, PKM2 knockout mice have a high incidence to develop hepatocellular carcinoma spontaneously after a long latency due to the imbalance in metabolism [57]. These results against the notion that PKM2 plays an oncogenic role in vivo.

\section{The continuing episode: is PKM2 a cancer biomarker and drug target?}

Although the results of genetically engineering mouse model do not support the tumor-promoting activity of PKM2, overexpression of PKM2 is universally found in human cancers and is associated with poor clinical outcome (Table 1) [58-81]. Two recent meta-analysis studies also supported this conclusion. Wu et al. analyzed the data of 2812 patients with solid tumors of digestive system obtained from 16 cohort studies and found that overexpression of PKM2 is associated with reduced overall survival in gastric cancer, esophageal squamous cell carcinoma, hepatocellular carcinoma, biliary cancer and oral cancer [82]. However, PKM2 is not a prognostic factor for pancreatic cancer. This finding is not consistent with previous studies showing that increase of PKM2 is an indicator of poor survival of pancreatic cancer patients $[71,73,77]$. Another investigation included 4796 cases from 27 individual studies demonstrated that PKM2 upregulation is correlated with worse overall survival, disease-free survival and recurrence-free survival in pooled data [83]. However, stratified by cancer type, PKM2 does not predict a poor survival of pancreatic cancer. Collectively, PKM2 seems to be a liable prognostic marker in most of solid tumors. 


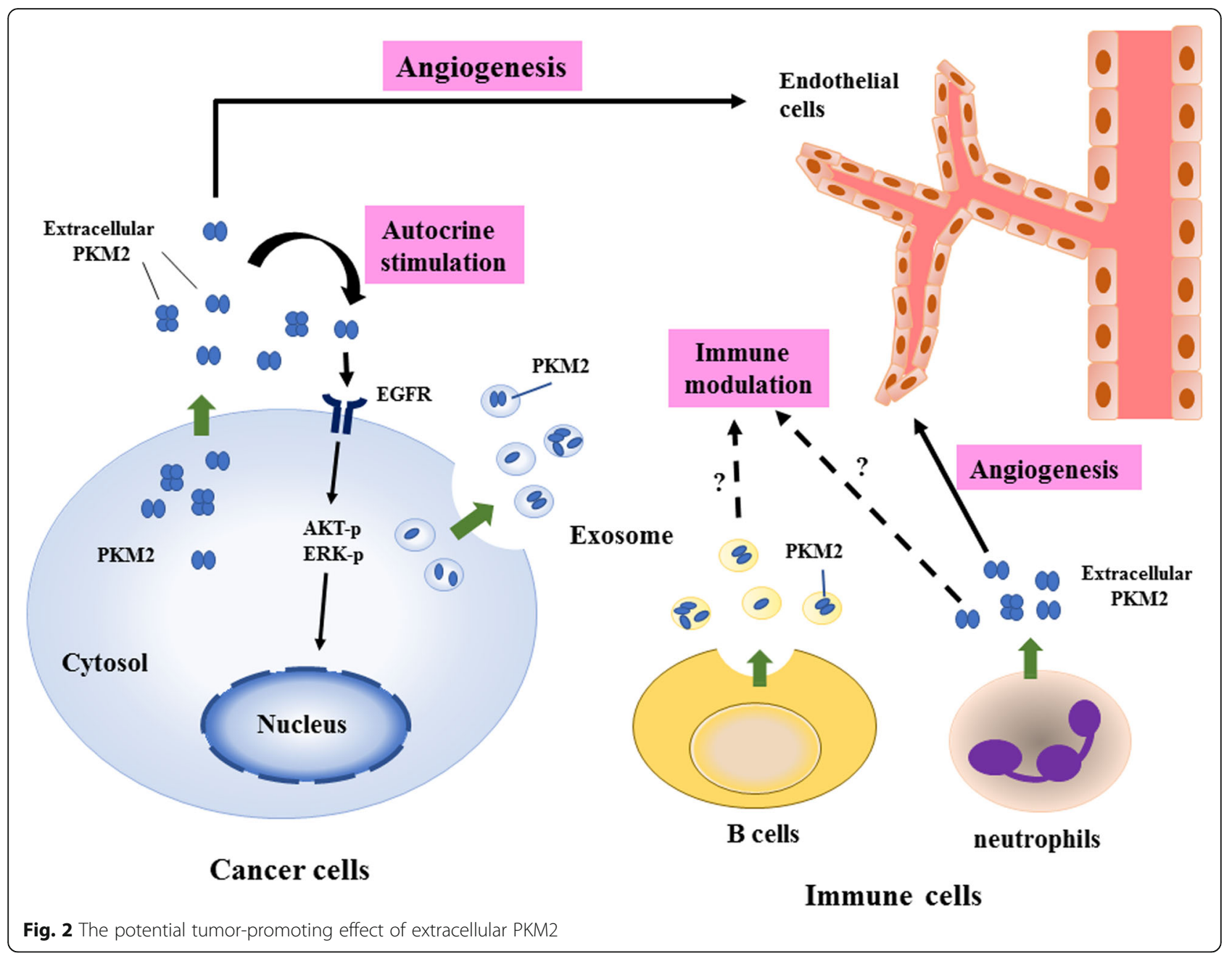

On the contrary, the use of PKM2 as a diagnostic factor is controversial. A proteomic analysis demonstrated that PKM2 is a potential diagnostic marker for the detection of lung cancer [84]. However, a recent study suggested PKM2 is not a good diagnostic marker for lung cancer due to low specificity [85]. Similarly, PKM2 alone is unlikely to be a useful marker for the screening of colon cancer [86]. However, combination of multiple markers could increase sensitivity and specificity for cancer diagnosis [86].

The therapeutic potential of PKM2 is an intriguing event in cancer treatment. From one side, inhibition of PKM2 is expected to inhibit glycolysis, impair gene transcription and suppress cellular proliferation. Therefore, PKM2 inhibitors seem to be good candidates for anti-cancer drug development. By using library screening, Vander Heiden et al. identified three novel classes of PKM2 inhibitors and showed that the most effective compound inhibited PKM2 activity and induced death of cancer cells [31]. Recently, Ning et al. found that novel naphthoquinone derivatives are potent PKM2 inhibitors [87]. One effective compound
$3 \mathrm{k}$ suppressed the proliferation of multiple cancer cell lines at sub-micromolar concentrations while it showed little detrimental effect on normal cells. From the other side, activation of PKM2 may also inhibit tumor growth. Because the low activity PKM2 dimer is the major isoform that triggers glycolysis in the cytoplasm and gene transcription in the nucleus in cancer cells, PKM2 activators which can promote the formation of tetrameric PKM2 may switch glycolysis to mitochondria pathway and reduce nuclear entry to attenuate gene transcription. Both effects impair metabolic demand and growth-supporting signaling that leads to tumor regression. Two pioneer studies identified various PKM2 activators and characterized their specificity in vitro [88, 89]. A subsequent study demonstrated that PKM2 activators indeed promoted tetramer formation and suppressed tumor growth in vivo [19]. These results suggested PKM2 activators could be promising anti-cancer drugs.

Resistance to chemotherapy is a major blockage for cancer treatment. Overcoming the inherent chemoresistance 
Table 1 Prognostic significance of PKM2 in human cancers

\begin{tabular}{|c|c|c|c|c|c|}
\hline Cancer & Detection & Gene & Expression & Prognosis & Ref. \\
\hline \multirow[t]{2}{*}{ Mesothelioma } & \multirow[t]{2}{*}{ qRT-PCR } & PKM2, ARHGDIA & \multirow[t]{2}{*}{ Increased } & \multirow[t]{2}{*}{ Poor } & \multirow[t]{2}{*}[58]{} \\
\hline & & TM4SF1, COBLL1 & & & \\
\hline Chordoma & $\mathrm{IHC}$ & PKM2, ENO1, gp96 & increased & poor & [59] \\
\hline Gastric cancer & $\mathrm{q}-\mathrm{RT}-\mathrm{PCR} / \mathrm{IHC}$ & PKM2 & increased & poor & [60] \\
\hline \multirow[t]{2}{*}{ Gallbladder cancer } & \multirow[t]{2}{*}{$\mathrm{IHC}$} & PKM2, ACVR 1C & \multirow[t]{2}{*}{ increased } & \multirow[t]{2}{*}{ poor } & \multirow[t]{2}{*}[61]{} \\
\hline & & GAPDHS, TYRP1 & & & \\
\hline Melanoma & $\mathrm{IHC}$ & PKM2, GAPDH & increased & poor & [62] \\
\hline Ovarian cancer & $\mathrm{q}-\mathrm{RT}$-PCR/IHC & PKM2, GAPDH, ATP5B & increased & poor & [63] \\
\hline Tongue cancer & $\mathrm{IHC}$ & PKM2, LDH5 & increased & poor & [64] \\
\hline Esophageal cancer & IHCMB & PKM2, HK1, PFKB & increased & poor & [65] \\
\hline Hepatoma & $\mathrm{IHC}$ & PKM2 & increased & poor & [66] \\
\hline Hepatoma & $\mathrm{IHC}$ & PKM2, TRIM35 & increased & poor & [67] \\
\hline Hepatoma & $\mathrm{IHC}$ & PKM2, Bim & increased & poor & [68] \\
\hline Cervical cancer & $\mathrm{IHC}$ & PKM2 & increased & poor & [69] \\
\hline Oral cancer & $\mathrm{IHC}$ & PKM2 & increased & poor & [70] \\
\hline Pancreatic cancer & $\mathrm{IHC}$ & PKM2, HK2 & increased & poor & [71] \\
\hline Breast cancer & $\mathrm{IHC}$ & PKM2, VEGF-C & increased & poor & [72] \\
\hline Pancreatic cancer & $\mathrm{IHC}$ & PKM2 & increased & poor & [73] \\
\hline Gastric cancer & $\mathrm{IHC}$ & PKM2, HK1 & increased & poor & [74] \\
\hline Cholangiocarcinoma & $\mathrm{IHC}$ & PKM2 & increased & poor & {$[75]$} \\
\hline Colorectal cancer & $\mathrm{IHC}$ & PKM2 & increased & poor & [76] \\
\hline Pancreatic cancer & $\mathrm{IHC}$ & PKM2, LDHA & increased & poor & [77] \\
\hline Gallbladder cancer & $\mathrm{IHC}$ & PKM2 & increased & poor & [78] \\
\hline Osteosarcoma & $\mathrm{IHC}$ & PKM2 & increased & poor & [79] \\
\hline Gastric cancer & $\mathrm{IHC}$ & PKM2 & increased & poor & [80] \\
\hline Ovarian cancer & $\mathrm{IHC}$ & PKM2 & increased & poor & [81] \\
\hline
\end{tabular}

Abbreviation: $q-R T-P C R$ quantitative reverse-transcriptase polymerase chain reaction, ARHGDI Rho GDP dissociation inhibitor alpha, TM4SF1 transmembrane $4 \mathrm{~L}$ six family member 1, COBLL1 Cordon-Bleu WH2 repeat protein like 1, ENO1 enolase-1, gp96 heat shock protein 90 beta family member 1, ACVR 1C activin A receptor type 1C, GAPDH glyceraldehyde-3-phosphate dehydrogenase, GAPDHS glyceraldehyde-3-phosphate dehydrogenase, spermatogenic, TYRP1 tyrosinase related protein 1, ATP5B mitochondrial ATP synthase beta subunit, LDH5 lactate dehydrogenase 5, HK1 hexokinase 1, PFKB phosphofructokinase-2, TRIM35 Tripartite motif containing $35, H K 2$ hexokinase 2, VEGF-C vascular endothelial growth factor $C$

of cancer cells is urgent for clinical research. The roles of PKM2 in chemoresistance of cancer cells have been revealed, and targeting PKM2 has been shown to resensitize chemoresistant cancer cells. A recent study showed that CD44 interacts with PKM2 and suppresses PKM2 activity via increasing Tyr105 phosphorylation of PKM2. CD44 ablation induced the switch from aerobic glycolysis to mitochondrial respiration and increasing reactive oxygen species (ROS) production, resulting in the enhancement of cisplatin sensitivity in colorectal cancer cells [90]. Inhibition of PKM2 activity was demonstrated to suppress glycolysis and overcome cisplatin resistance. Therefore, the combination of cisplatin and PKM2 inhibitors may be an effective strategy for chemotherapy (Fig. 3). PKM2 has also been reported to participate in the regulation of gemcitabine resistance in pancreatic cancer cells. Kim et al. showed that PKM2 promotes gemcitabine resistance through negatively regulating p38-mediated p53 phosphorylation, therefore reducing transcriptional activity of p53 and suppressing the expression of pro-apoptotic genes [91]. Moreover, ectopic expression of R399E-PKM2, which preferentially forms dimeric PKM2, enhances the resistance of pancreatic cancer cells to gemcitabine. These evidences suggest PKM2 contributes to the induction of drug resistance via a non-metabolic mechanism. Because dimeric PKM2 acts mainly as a transcriptional regulator in cancer cells, PKM2 activators which promote the formation of tetrameric PKM2 may be effective in suppressing non-metabolic function of PKM2 and reducing resistance to gemcitabine in pancreatic cancer cells. Nuclear PKM2 has also been demonstrated to contribute to the resistance of EGFR inhibitor in colorectal cancer and lung cancer [92, 93]. Li et al. showed that nuclear PKM2mediated STAT3 phosphorylation reduces the sensitivity 


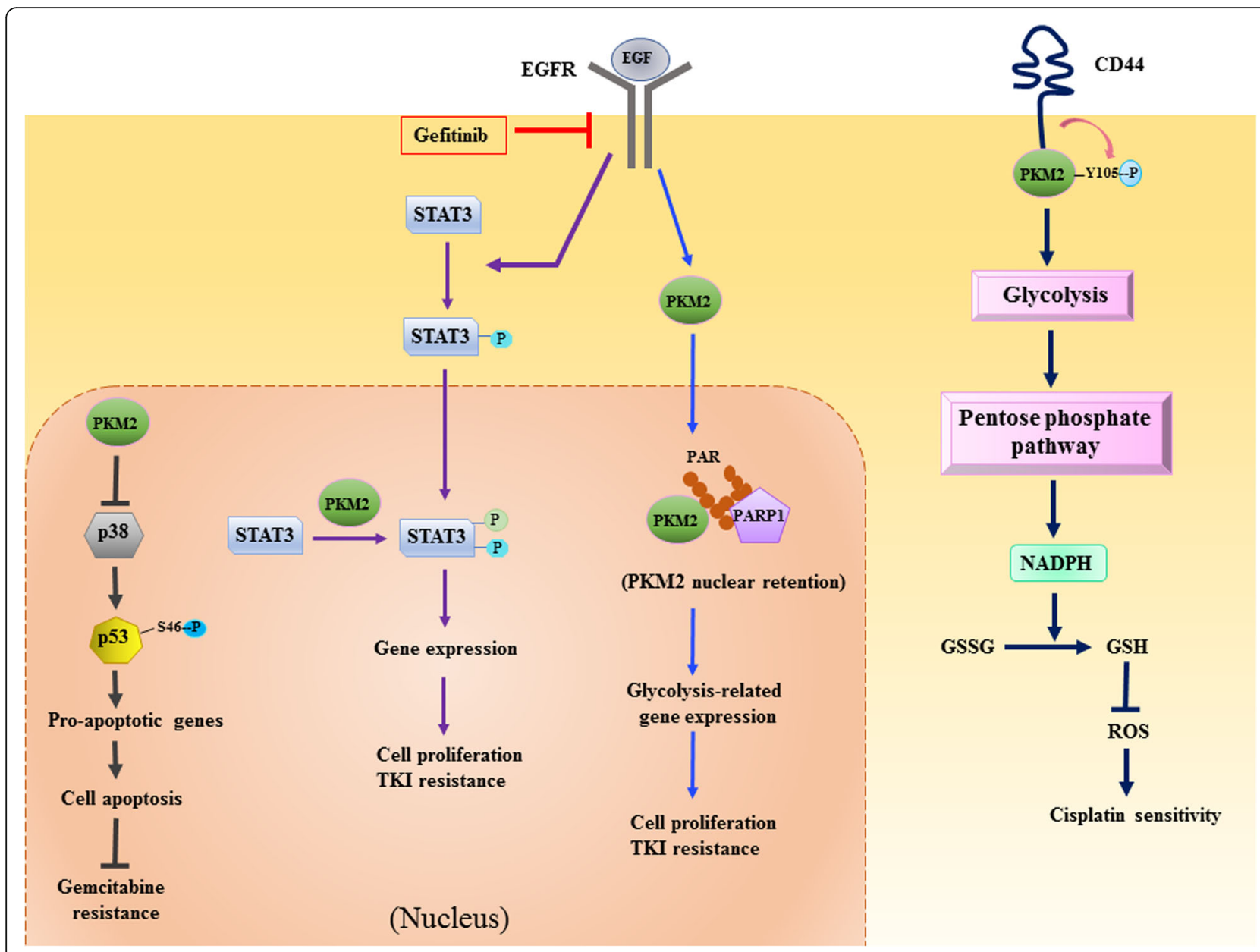

Fig. 3 PKM2 contributes to the proliferation and drug resistance in cancer cells

of colorectal cancer cells to gefitinib and disruption of the interaction of nuclear PKM2 and STAT3 restored gefitinib sensitivity in the cells [92]. More recently, another study demonstrated that PKM2 translocates into the nucleus and interacts with poly-ADP ribose (PAR) upon growth factor stimulation. The PAR-binding activity of PKM2 is critical for nuclear retention and gene transcription of PKM2 and is important for the promotion of cell proliferation and tumor growth. They concluded that inhibition of PKM2 nuclear function may overcome the resistance of EGFR-mutated cancer cells [93]. Both studies pointed out a crucial role of nuclear PKM2 in mediating drug resistance in cancers and suggested targeting nuclear PKM2 may be a promising strategy to override the resistance.

\section{Conclusions}

Our understanding of PKM2 function expands dramatically in the past two decades. Originally identified as a metabolic enzyme in glycolysis, PKM2 is now found to be a multi-face protein that fuels different aspects of cancer cells to sustain tumor growth. The use of PKM2 as a prognostic marker has been validated in a variety of cancers. In addition, the application of PKM2 activators or inhibitors in cancer therapy can be expected in the coming decade.

\section{Acknowledgments}

This study was supported by the grant: CA-106-PP-15 and CA-107-PP-14 from the Ministry of Health and Welfare, Taiwan, Republic of China.

\section{Authors' contributions}

$\mathrm{MCH}$ and $\mathrm{WCH}$ wrote the manuscript. Both authors read and approved the final manuscript.

\section{Competing interests}

The authors declare that they have no competing interests.

\section{Publisher's Note}

Springer Nature remains neutral with regard to jurisdictional claims in published maps and institutional affiliations.

Received: 11 October 2017 Accepted: 1 February 2018

Published online: 19 February 2018

\section{References}

1. Cori CF. Embden and the Glycolytic pathway. Trends Biochem Sci. 1983;8:257-9. 
2. Cori CF. Some highlights of the early period of bioenergetics. Mol Cell Biochem. 1974;5:47-53.

3. Dayton TL, Jacks T, Vander Heiden MG. PKM2, cancer metabolism, and the road ahead. EMBO Rep. 2016;17:1721-30.

4. Barnett JA. A history of research on yeasts 5: the fermentation pathway. Yeast. 2003;20:509-43.

5. Tanaka T, Harano Y, Morimura H, Mori R. Evidence for the presence of two types of pyruvate kinase in rat liver. Biochem Biophys Res Commun. 1965;21:55-60.

6. Tanaka T, Harano Y, Sue F, Morimura H. Crystallization, characterization and metabolic regulation of two types of pyruvate kinase isolated from rat tissues. J Biochem. 1967;62:71-91.

7. Susor WA, Rutter WJ. Some distinctive properties of pyruvate kinase purified from rat liver. Biochem Biophys Res Commun. 1968;30:14-20.

8. Osterman J, Fritz PJ. Pyruvate kinase isozymes: a comparative study in tissues of various mammalian species. Comp Biochem Physiol B. 1973; 44:1077-85

9. Burke RL, Tekamp-Olson P, Najarian R. The isolation, characterization, and sequence of the pyruvate kinase gene of Saccharomyces Cerevisiae. J Biol Chem. 1983;258:2193-201.

10. Lonberg N, Gilbert W. Primary structure of chicken muscle pyruvate kinase mRNA. Proc Natl Acad Sci U S A. 1983;80:3661-5.

11. Simon MP, Besmond C, Cottreau D, Weber A, Chaumet-Riffaud P, Dreyfus JC, Trepat JS, Marie J, Kahn A. Molecular cloning of cDNA for rat L-type pyruvate kinase and aldolase B. J Biol Chem. 1983;258:14576-84.

12. Noguchi T, Inoue H, Tanaka T. The M1- and M2-type isozymes of rat pyruvate kinase are produced from the same gene by alternative RNA splicing. J Biol Chem. 1986;261:13807-12.

13. Takenaka M, Noguchi T, Sadahiro S, Hirai H, Yamada K, Matsuda T, Imai E, Tanaka T. Isolation and characterization of the human pyruvate kinase M gene. Eur J Biochem. 1991;198:101-6.

14. Christofk HR, Vander Heiden MG, Harris MH, Ramanathan A, Gerszten RE, Wei R, Fleming MD, Schreiber SL, Cantley LC. The M2 splice isoform of pyruvate kinase is important for cancer metabolism and tumour growth. Nature. 2008:452:230-3.

15. Mazurek S. Pyruvate kinase type M2: a key regulator of the metabolic budget system in tumor cells. Int J Biochem Cell Biol. 2011;43:969-80.

16. Hacker HJ, Steinberg $P$, Bannasch P. Pyruvate kinase isoenzyme shift from L-type to M2-type is a late event in hepatocarcinogenesis induced in rats by a choline-deficient/DL-ethionine-supplemented diet. Carcinogenesis. 1998;19:99-107.

17. Iqbal MA, Gupta V, Gopinath P, Mazurek S, Bamezai RN. Pyruvate kinase M2 and cancer: an updated assessment. FEBS Lett. 2014;588:2685-92.

18. David CJ, Chen M, Assanah M, Canoll P, Manley JL. HnRNP proteins controlled by c-Myc deregulate pyruvate kinase mRNA splicing in cancer. Nature. 2010;463:364-8.

19. Anastasiou D, Yu Y, Israelsen WJ, Jiang JK, Boxer MB, Hong BS, Tempel W, Dimov S, Shen M, Jha A, et al. Pyruvate kinase M2 activators promote tetramer formation and suppress tumorigenesis. Nat Chem Biol. 2012;8:839-47.

20. Spoden GA, Mazurek S, Morandell D, Bacher N, Ausserlechner MJ, JansenDurr $P$, Eigenbrodt E, Zwerschke W. Isotype-specific inhibitors of the glycolytic key regulator pyruvate kinase subtype M2 moderately decelerate tumor cell proliferation. Int J Cancer. 2008;123:312-21.

21. Porporato PE, Dhup S, Dadhich RK, Copetti T, Sonveaux P. Anticancer targets in the glycolytic metabolism of tumors: a comprehensive review. Front Pharmacol. 2011;2:49.

22. $\mathrm{Li} Z$ Z, Yang $\mathrm{P}, \mathrm{Li}$ Z. The multifaceted regulation and functions of PKM2 in tumor progression. Biochim Biophys Acta. 1846;2014:285-96.

23. Israelsen WJ, Vander Heiden MG. Pyruvate kinase: function, regulation and role in cancer. Semin Cell Dev Biol. 2015;43:43-51.

24. Dong G, Mao Q, Xia W, Xu Y, Wang J, Xu L, Jiang F. PKM2 and cancer: the function of PKM2 beyond glycolysis. Oncol Lett. 2016;11:1980-6.

25. Jurica MS, Mesecar A, Heath PJ, Shi W, Nowak T, Stoddard BL. The allosteric regulation of pyruvate kinase by fructose-1,6-bisphosphate. Structure. 1998;6:195-210.

26. Keller KE, Tan IS, Lee YS. SAICAR stimulates pyruvate kinase isoform M2 and promotes cancer cell survival in glucose-limited conditions. Science. 2012; 338:1069-72.

27. Chaneton B, Hillmann P, Zheng L, Martin ACL, Maddocks ODK, Chokkathukalam A, Coyle JE, Jankevics A, Holding FP, Vousden KH, et al. Serine is a natural ligand and allosteric activator of pyruvate kinase M2. Nature. 2012;491:458-62.
28. Vijayvargiya R, Schwark WS, Singhal RL. Pyruvate kinase: modulation by L -phenylalanine and L -alanine. Can J Biochem. 1969;47:895-8.

29. Weber $\mathrm{G}$. Inhibition of human brain pyruvate kinase and hexokinase by phenylalanine and phenylpyruvate: possible relevance to phenylketonuric brain damage. Proc Natl Acad Sci U S A. 1969;63:1365-9.

30. Palsson-McDermott EM, Curtis AM, Goel G, Lauterbach MA, Sheedy FJ, Gleeson LE, van den Bosch MW, Quinn SR, Domingo-Fernandez R, Johnston $D G$, et al. Pyruvate kinase $M 2$ regulates Hif-1alpha activity and IL-1 beta induction and is a critical determinant of the warburg effect in LPSactivated macrophages. Cell Metab. 2015;21:65-80.

31. Vander Heiden MG, Christofk HR, Schuman E, Subtelny AO, Sharfi H, Harlow EE, Xian J, Cantley LC. Identification of small molecule inhibitors of pyruvate kinase M2. Biochem Pharmacol. 2010;79:1118-24.

32. Mazurek S, Drexler HC, Troppmair J, Eigenbrodt E, Rapp UR. Regulation of pyruvate kinase type M2 by A-Raf: a possible glycolytic stop or go mechanism. Anticancer Res. 2007;27:3963-71.

33. Hitosugi T, Kang S, Vander Heiden MG, Chung TW, Elf S, Lythgoe K, Dong S, Lonial S, Wang X, Chen GZ, et al. Tyrosine phosphorylation inhibits PKM2 to promote the Warburg effect and tumor growth. Sci Signal. 2009;2:ra73.

34. Christofk HR, Vander Heiden MG, Wu N, Asara JM, Cantley LC. Pyruvate kinase M2 is a phosphotyrosine-binding protein. Nature. 2008;452:181-6.

35. Mukherjee J, Ohba S, See WL, Phillips JJ, Molinaro AM, Pieper RO. PKM2 uses control of HuR localization to regulate p27 and cell cycle progression in human glioblastoma cells. Int J Cancer. 2016;139:99-111.

36. Huang L, Yu Z, Zhang Z, Ma W, Song S, Huang G. Interaction with Pyruvate Kinase M2 destabilizes Tristetraprolin by Proteasome degradation and regulates cell proliferation in breast cancer. Sci Rep. 2016;6:22449.

37. Liang J, Cao R, Wang X, Zhang Y, Wang P, Gao H, Li C, Yang F, Zeng R, Wei $P$, et al. Mitochondrial PKM2 regulates oxidative stress-induced apoptosis by stabilizing Bcl2. Cell Res. 2017;27:329-51.

38. Yang W, Xia Y, Ji H, Zheng Y, Liang J, Huang W, Gao X, Aldape K, Lu Z. Nuclear PKM2 regulates beta-catenin transactivation upon EGFR activation. Nature. 2011:480:118-22

39. Gao X, Wang H, Yang JJ, Liu X, Liu ZR. Pyruvate kinase M2 regulates gene transcription by acting as a protein kinase. Mol Cell. 2012;45:598-609.

40. Jiang $Y$, Wang $Y$, Wang T, Hawke DH, Zheng Y, Li X, Zhou Q, Majumder S, Bi E, Liu DX, et al. PKM2 phosphorylates MLC2 and regulates cytokinesis of tumour cells. Nat Commun. 2014:5:5566.

41. Jiang Y, Li X, Yang W, Hawke DH, Zheng Y, Xia Y, Aldape K, Wei C, Guo F, Chen $Y$, Lu Z. PKM2 regulates chromosome segregation and mitosis progression of tumor cells. Mol Cell. 2014;53:75-87.

42. Keller KE, Doctor ZM, Dwyer ZW, Lee YS. SAICAR induces protein kinase activity of PKM2 that is necessary for sustained proliferative signaling of cancer cells. Mol Cell. 2014;53:700-9.

43. Morfouace M, Lalier L, Oliver L, Cheray M, Pecqueur C, Cartron PF, Vallette FM. Control of glioma cell death and differentiation by PKM2-Oct4 interaction. Cell Death Dis. 2014;5:e1036.

44. Azoitei N, Becher A, Steinestel K, Rouhi A, Diepold K, Genze F, Simmet T, Seufferlein T. PKM2 promotes tumor angiogenesis by regulating HIF-1alpha through NF-kappaB activation. Mol Cancer. 2016;15:3.

45. Hosios AM, Fiske BP, Gui DY, Vander Heiden MG. Lack of evidence for PKM2 protein Kinase activity. Mol Cell. 2015;59:850-7.

46. Buschow SI, van Balkom BW, Aalberts M, Heck AJ, Wauben M, Stoorvogel W. MHC class II-associated proteins in B-cell exosomes and potential functional implications for exosome biogenesis. Immunol Cell Biol. 2010;88:851-6.

47. Mathivanan S, Ji H, Simpson RJ. Exosomes: extracellular organelles important in intercellular communication. J Proteome. 2010;73:1907-20.

48. Kim DK, Kang B, Kim OY, Choi DS, Lee J, Kim SR, Go G, Yoon YJ, Kim $J$, Jang SC, et al. EVpedia: an integrated database of high-throughput data for systemic analyses of extracellular vesicles. J Extracell Vesicles. 2013:2:20384.

49. Mathivanan S, Simpson RJ. ExoCarta: a compendium of exosomal proteins and RNA. Proteomics. 2009;9:4997-5000.

50. Thery C, Zitvogel L, Amigorena S. Exosomes: composition, biogenesis and function. Nat Rev Immunol. 2002;2:569-79.

51. Raposo G, Stoorvogel W. Extracellular vesicles: exosomes, microvesicles, and friends. J Cell Biol. 2013;200:373-83.

52. Li L, Zhang Y, Qiao J, Yang JJ, Liu ZR. Pyruvate kinase M2 in blood circulation facilitates tumor growth by promoting angiogenesis. J Biol Chem. 2014;289:25812-21. 
53. Yang $P, L i Z$, Wang $Y$, Zhang $L, W u H, L i Z$. Secreted pyruvate kinase $M 2$ facilitates cell migration via PI3KJAkt and Wnt/beta-catenin pathway in colon cancer cells. Biochem Biophys Res Commun. 2015;459:327-32.

54. Zhang Y, Li L, Liu Y, Liu ZR. PKM2 released by neutrophils at wound site facilitates early wound healing by promoting angiogenesis. Wound Repair Regen. 2016;24:328-36.

55. Hsu MC, Hung WC, Yamaguchi H, Lim SO, Liao HW, Tsai CH, Hung MC. Extracellular PKM2 induces cancer proliferation by activating the EGFR signaling pathway. Am J Cancer Res. 2016;6:628-38.

56. Israelsen WJ, Dayton TL, Davidson SM, Fiske BP, Hosios AM, Bellinger G, Li J, Yu Y, Sasaki M, Horner JW, et al. PKM2 isoform-specific deletion reveals a differential requirement for pyruvate kinase in tumor cells. Cell. 2013;155:397-409.

57. Dayton TL, Gocheva V, Miller KM, Israelsen WJ, Bhutkar A, Clish CB, Davidson SM, Luengo A, Bronson RT, Jacks T, Vander Heiden MG. Germline loss of PKM2 promotes metabolic distress and hepatocellular carcinoma. Genes Dev. 2016;30:1020-33.

58. Gordon GJ, Dong L, Yeap BY, Richards WG, Glickman JN, Edenfield H, Mani M, Colquitt R, Maulik G, Van Oss B, et al. Four-gene expression ratio test for survival in patients undergoing surgery for mesothelioma. J Natl Cancer Inst. 2009;101:678-86

59. Zhou H, Chen CB, Lan J, Liu C, Liu XG, Jiang L, Wei F, Ma QJ, Dang GT, Liu ZJ. Differential proteomic profiling of chordomas and analysis of prognostic factors. J Surg Oncol. 2010;102:720-7.

60. Lim JY, Yoon SO, Seol SY, Hong SW, Kim JW, Choi SH, Cho JY. Overexpression of the M2 isoform of pyruvate kinase is an adverse prognostic factor for signet ring cell gastric cancer. World J Gastroenterol. 2012;18:4037-43.

61. Li J, Yang Z, Zou Q, Yuan Y, Li J, Liang L, Zeng G, Chen S. PKM2 and ACVR IC are prognostic markers for poor prognosis of gallbladder cancer. Clin Transl Oncol. 2014;16:200-7.

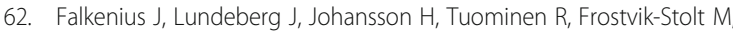
Hansson J, Egyhazi Brage S. High expression of glycolytic and pigment proteins is associated with worse clinical outcome in stage III melanoma. Melanoma Res. 2013;23:452-60

63. Hjerpe E, Egyhazi Brage S, Carlson J, Frostvik Stolt M, Schedvins K, Johansson H, Shoshan M, Avall-Lundqvist E. Metabolic markers GAPDH, PKM2, ATP5B and BEC-index in advanced serous ovarian cancer. BMC Clin Pathol. 2013;13:30

64. Yuan C, Li Z, Wang Y, Qi B, Zhang W, Ye J, Wu H, Jiang H, Song LN, Yang J, Cheng J. Overexpression of metabolic markers PKM2 and LDH5 correlates with aggressive clinicopathological features and adverse patient prognosis in tongue cancer. Histopathology. 2014;65:595-605.

65. Li W, Xu Z, Hong J, Xu Y. Expression patterns of three regulation enzymes in glycolysis in esophageal squamous cell carcinoma: association with survival. Med Oncol. 2014;31:118.

66. Liu WR, Tian MX, Yang LX, Lin YL, Jin L, Ding ZB, Shen YH, Peng YF, Gao $\mathrm{DM}$, Zhou J, et al. PKM2 promotes metastasis by recruiting myeloid-derived suppressor cells and indicates poor prognosis for hepatocellular carcinoma. Oncotarget. 2015;6:846-61.

67. Chen Z, Lu X, Wang Z, Jin G, Wang Q, Chen D, Chen T, Li J, Fan J, Cong W, et al. Co-expression of PKM2 and TRIM35 predicts survival and recurrence in hepatocellular carcinoma. Oncotarget. 2015;6:2538-48.

68. Hu W, Lu SX, Li M, Zhang C, Liu LL, Fu J, Jin JT, Luo RZ, Zhang CZ, Yun JP. Pyruvate kinase M2 prevents apoptosis via modulating Bim stability and associates with poor outcome in hepatocellular carcinoma. Oncotarget. 2015;6:6570-83.

69. Zhao Y, Shen L, Chen X, Qian Y, Zhou Q, Wang Y, Li K, Liu M, Zhang S, Huang $X$. High expression of PKM2 as a poor prognosis indicator is associated with radiation resistance in cervical cancer. Histol Histopathol. 2015;30:1313-20.

70. Wang Y, Zhang X, Zhang Y, Zhu Y, Yuan C, Qi B, Zhang W, Wang D, Ding X, $\mathrm{Wu} \mathrm{H}$, Cheng J. Overexpression of pyruvate kinase M2 associates with aggressive clinicopathological features and unfavorable prognosis in oral squamous cell carcinoma. Cancer Biol Ther. 2015;16:839-45.

71. Ogawa H, Nagano H, Konno M, Eguchi H, Koseki J, Kawamoto K, Nishida N, Colvin $\mathrm{H}$, Tomokuni A, Tomimaru Y, et al. The combination of the expression of hexokinase 2 and pyruvate kinase M2 is a prognostic marker in patients with pancreatic cancer. Mol Clin Oncol. 2015;3:563-71.

72. Lin Y, Liu F, Fan Y, Qian X, Lang R, Gu F, Gu J, Fu L. Both high expression of pyruvate kinase $\mathrm{M} 2$ and vascular endothelial growth factor-C predicts poorer prognosis in human breast cancer. Int I Clin Exp Pathol. 2015;8:8028-37.

73. Lockney NA, Zhang M, Lu Y, Sopha SC, Washington MK, Merchant N, Zhao Z, Shyr Y, Chakravarthy AB, Xia F. Pyruvate Kinase muscle Isoenzyme 2
(PKM2) expression is associated with overall survival in pancreatic Ductal Adenocarcinoma. J Gastrointest Cancer. 2015:46:390-8.

74. Gao Y, Xu D, Yu G, Liang J. Overexpression of metabolic markers HK1 and PKM2 contributes to lymphatic metastasis and adverse prognosis in Chinese gastric cancer. Int J Clin Exp Pathol. 2015;8:9264-71.

75. Yu G, Yu W, Jin G, Xu D, Chen Y, Xia T, Yu A, Fang W, Zhang X, Li Z, Xie K. PKM2 regulates neural invasion of and predicts poor prognosis for human hilar cholangiocarcinoma. Mol Cancer. 2015;14:193.

76. Cui R, Shi XY. Expression of pyruvate kinase M2 in human colorectal cancer and its prognostic value. Int J Clin Exp Pathol. 2015;8:11393-9.

77. Mohammad GH, Olde Damink SW, Malago M, Dhar DK, Pereira SP. Pyruvate Kinase M2 and lactate Dehydrogenase a are Overexpressed in pancreatic cancer and correlate with poor outcome. PLoS One. 2016;11:e0151635.

78. Lu W, Cao Y, Zhang Y, Li S, Gao J, Wang XA, Mu J, Hu YP, Jiang L, Dong P, et al. Up-regulation of PKM2 promote malignancy and related to adverse prognostic risk factor in human gallbladder cancer. Sci Rep. 2016;6:26351.

79. Liu ZX, Hong L, Fang SQ, Tan GH, Huang PG, Zeng Z, Xia X, Wang XX. Overexpression of pyruvate kinase M2 predicts a poor prognosis for patients with osteosarcoma. Tumour Biol. 2016;37:14923-8.

80. Wang C, Jiang J, Ji J, Cai Q, Chen X, Yu Y, Zhu Z, Zhang J. PKM2 promotes cell migration and inhibits autophagy by mediating PISK/AKT activation and contributes to the malignant development of gastric cancer. Sci Rep. 2017;7:2886

81. Chao TK, Huang TS, Liao YP, Huang RL, Su PH, Shen HY, Lai HC, Wang YC. Pyruvate kinase M2 is a poor prognostic marker of and a therapeutic target in ovarian cancer. PLoS One. 2017;12:e0182166.

82. Wu J, Hu L, Chen M, Cao W, Chen H, He T. Pyruvate kinase M2 overexpression and poor prognosis in solid tumors of digestive system: evidence from 16 cohort studies. Onco Targets Ther. 2016;9:4277-88.

83. Zhu H, Luo H, Zhu X, Hu X, Zheng L, Zhu X. Pyruvate kinase M2 (PKM2) expression correlates with prognosis in solid cancers: a meta-analysis. Oncotarget. 2017;8:1628-40

84. Peng XC, Gong FM, Zhao YW, Zhou LX, Xie YW, Liao HL, Lin HJ, Li ZY, Tang $\mathrm{MH}$, Tong AP. Comparative proteomic approach identifies PKM2 and cofilin1 as potential diagnostic, prognostic and therapeutic targets for pulmonary adenocarcinoma. PLoS One. 2011;6:e27309.

85. Rzechonek A, Kaminska A, Mamczur P, Drapiewski A, Budzynski W. Limited clinical significance of Dimeric form of Pyruvate Kinase as a diagnostic and prognostic biomarker in non-small cell lung cancer. Adv Exp Med Biol. 2017;955:51-7.

86. Fung KY, Tabor B, Buckley MJ, Priebe IK, Purins L, Pompeia C, Brierley GV, Lockett T, Gibbs P, Tie J, et al. Blood-based protein biomarker panel for the detection of colorectal cancer. PLoS One. 2015;10:e0120425.

87. Ning $X$, Qi H, Li R, Li Y, Jin Y, McNutt MA, Liu J, Yin Y. Discovery of novel naphthoquinone derivatives as inhibitors of the tumor cell specific M2 isoform of pyruvate kinase. Eur J Med Chem. 2017;138:343-52.

88. Boxer MB, Jiang JK, Vander Heiden MG, Shen M, Skoumbourdis AP, Southall $N$, Veith H, Leister W, Austin CP, Park HW, et al. Evaluation of substituted $\mathrm{N}, \mathrm{N}^{\prime}$-diarylsulfonamides as activators of the tumor cell specific M2 isoform of pyruvate kinase. J Med Chem. 2010;53:1048-55.

89. Jiang JK, Boxer MB, Vander Heiden MG, Shen M, Skoumbourdis AP, Southall $\mathrm{N}$, Veith $\mathrm{H}$, Leister W, Austin CP, Park HW, et al. Evaluation of thieno[3,2b]pyrrole[3,2-d]pyridazinones as activators of the tumor cell specific M2 isoform of pyruvate kinase. Bioorg Med Chem Lett. 2010;20:3387-93.

90. Tamada M, Nagano O, Tateyama S, Ohmura M, Yae T, Ishimoto T, Sugihara E, Onishi N, Yamamoto T, Yanagawa H, et al. Modulation of glucose metabolism by CD44 contributes to antioxidant status and drug resistance in cancer cells. Cancer Res. 2012;72:1438-48.

91. Kim DJ, Park YS, Kang MG, You YM, Jung Y, Koo H, Kim JA, Kim MJ, Hong $\mathrm{SM}$, Lee $\mathrm{KB}$, et al. Pyruvate kinase isoenzyme M2 is a therapeutic target of gemcitabine-resistant pancreatic cancer cells. Exp Cell Res. 2015;336:119-29.

92. Li Q, Zhang D, Chen X, He L, Li T, Xu X, Li M. Nuclear PKM2 contributes to gefitinib resistance via upregulation of STAT3 activation in colorectal cancer. Sci Rep. 2015;5:16082.

93. Li N, Feng L, Liu H, Wang J, Kasembeli M, Tran MK, Tweardy DJ, Lin SH, Chen J. PARP inhibition suppresses growth of EGFR-mutant cancers by targeting nuclear PKM2. Cell Rep. 2016;15:843-56. 